TITLE:

\title{
Multimarket contact under demand fluctuations
}

\author{
$\operatorname{AUTHOR}(\mathrm{S})$ :
}

Sekiguchi, Tadashi

\section{CITATION:}

Sekiguchi, Tadashi. Multimarket contact under demand fluctuations. International Journal of Game Theory 2015, 44(4): 1033-1048

ISSUE DATE:

2015-11

URL:

http://hdl.handle.net/2433/207640

\section{RIGHT:}

The final publication is available at Springer via http://dx.doi.org/10.1007/s00182-015-0464-z: The full-text file will be made open to the public on 1 November 2016 in accordance with publisher's 'Terms and Conditions for Self-Archiving'.; この論文は出版社版でありません。引用の際には出版社版をご確認ご利用ください。; This is not the published version. Please cite only the published version. 


\title{
Multimarket Contact under Demand Fluctuations*
}

\author{
Tadashi Sekiguchi ${ }^{\dagger}$ \\ Kyoto University
}

October 17, 2014

\begin{abstract}
The present paper studies repeated Bertrand oligopoly with multiple markets. The markets are subject to independent, stochastic fluctuations in demands. According to the literature, the demand fluctuations generally hinder collusion, while the multimarket contact sometimes facilitates it. We show that when only partial collusion is sustainable under a single market, the per-market expected profit under the most collusive equilibrium increases with the number of markets. Further, the difference between the total expected profit under full collusion and that under the most collusive equilibrium vanishes, if the number of markets goes to infinity. Thus the collusiondeterrence effects of fluctuated demands completely disappear in the limit.
\end{abstract}

JEL Classification: C72, C73, D43, L13.

Keywords: collusion, demand fluctuations, multimarket contact, repeated games

*An early version of the present paper has been circulated as Sekiguchi [6]. The author thanks Makoto Hanazono, Jin Li, Katsunori Ohta, Michael Powell, Dan Sasaki, Giulio Seccia, and two anonymous referees for very helpful comments. Financial supports from the Murata Science Foundation, the Tokyo Center for Economic Research, and the Grants-in-Aid for Scientific Research (20530153, 23000001) are gratefully acknowledged.

${ }^{\dagger}$ Institute of Economic Research, Kyoto University, Yoshida-Honmachi, Sakyo-ku, Kyoto 606-8501, Japan; sekiguchi@kier.kyoto-u.ac.jp. 


\section{Introduction}

The present paper studies a model of repeated Bertrand oligopoly, highlighting two typical features colluding firms often confront. First, they simultaneously interact in two or more markets (multimarket contact). Second, the demands in those markets are subject to random shocks (demand fluctuations). For instance, large nationwide firms often compete in many local markets simultaneously, and each local market is affected with idiosyncratic demand shocks. Another example is conglomerates competing over several industries, each of which has its own demand shocks.

In a framework of infinitely repeated games, existing results have clarified whether each of the two features facilitates or hinders formation of cartels. First, Bernheim and Whinston [1] point out that the multimarket contact never hinders collusion and sometimes facilitates it. In contrast, demand fluctuations generally hinder collusion, as Rotemberg and Saloner [5] show. The main purpose of the present paper is to examine how these two conflicting forces interact and affect the firms' ability to collude.

The present paper sets up a model of infinitely repeated games with symmetric and stochastic stage payoffs. The stage payoffs are i.i.d. over time. At the beginning of each period the players learn the stage payoffs of that period; all future payoffs are unknown at that time. This formulation of payoff fluctuations follows Rotemberg and Saloner [5]. While our model includes first-price auctions and moral hazard in teams as examples, we mainly apply it to Bertrand oligopoly and its multimarket version.

We focus on the most cooperative equilibrium (MCE); namely, the equilibrium which attains the greatest expected payoff among all symmetric subgame perfect equilibria of this repeated game. We start with a characterization of the MCE expected payoff, which we call the most cooperative payoff (MCP), for any level of discounting, which we utilize in examining the effects of multimarket contact under fluctuated demands.

Our main results are summarized as follows. Fix the probability distribution of stage payoffs. Then two threshold discount factors exist, $\bar{\delta}$ and $\underline{\delta}$ with $\bar{\delta}>\underline{\delta}$, such that regardless of the number of markets, (i) if $\delta<\underline{\delta}$, the MCE is repeated play of a static equilibrium, and (ii) if $\delta \geq \bar{\delta}$, the MCE attains full collusion. These are two polar cases where multimarket contact is irrelevant. Next, fix $\delta \in(\underline{\delta}, \bar{\delta})$. Then we show that for any $M$, the per-market MCP under $M$ markets is not greater than that under $M+1$ markets, and they are equal only in rare cases to be explained later. That is, on this range of discount factors, adding one more market almost always improves the per-market MCP.

Further, we show that the difference between the total expected profit under full collusion and the MCP converges to zero, if the number of markets goes to infinity. Hence, for relatively patient firms, the collusion-deterrence effects of fluctuated demands completely vanish in the limit. ${ }^{1}$ Another interpretation is that if the firms compete in a large number of markets, demand fluctuations do not much affect their ability to collude.

We are not the first to study the effect of multimarket contact under demand fluctuations. Bernheim and Whinston [1] have already studied the case of two markets, and show that the multimarket contact in general increases the per-market expected profit in comparison with the case of one market. In contrast, we consider an arbitrary number of

\footnotetext{
${ }^{1}$ The result does not entirely deny the collusion-deterrence effects of demand fluctuations in the following two senses. First, if $\underline{\delta} \leq \delta<\bar{\delta}$, full collusion cannot be sustained under any number of markets. Second, the limit result does not hold at $\underline{\delta}$.
} 
markets and verify that any possible profit loss due to demand fluctuations goes to zero when the number of markets goes to infinity.

An independent work by Li and Powell [3] is most closely related to the present paper. [3] also considers repeated games where multiple identical games with fluctuating payoffs are simultaneously played, and obtains a similar limit result. Both papers assume similar payoff structure, but the one in [3] is more general. A major difference is that their limit result is about equilibrium payoffs per component game, while ours examines equilibrium total payoffs and it is therefore stronger as a limit result. ${ }^{2}$

One important assumption in our setup is perfect monitoring; the players can directly observe their past actions. This assumption considerably simplifies analysis, enabling us to fully characterize the MCP. A more realistic assumption would be that the players only receive a noisy signal of their actions (imperfect monitoring). Matsushima [4] and Kobayashi and Ohta [2] investigate the effect of multimarket contact under imperfect monitoring, especially its role in promoting cooperation and/or collusion. ${ }^{3}$ [2] derives the most cooperative symmetric equilibrium payoff for any number of markets, assuming that the players are sufficiently patient. We rather consider the case of impatient players. [4] deals with the case of heavy discounting and is concerned with a limit result for the permarket profit. We instead consider any number of markets, and our limit result is for the total profit.

The rest of the present paper is organized as follows. Section 2 introduces the model. Section 3 characterizes the MCP. Section 4 applies the result in the previous section and studies effects of multimarket contact under demand fluctuations.

\section{Model}

Two players play a given normal-form game every period. ${ }^{4}$ Each player has an identical set of stage actions, denoted by $X$. Their stage payoff depends not only on the action pair selected in the period, denoted by $\left(x_{1}, x_{2}\right) \in X \times X$, but also on the state of that period. The set of possible states has $M+1$ elements, and we call them state 0 , state 1 , $\ldots$, state $M . u_{i}\left(x_{1}, x_{2}, k\right)$ denotes player $i$ 's stage payoff of the action pair $\left(x_{1}, x_{2}\right)$ under state $k$. We assume symmetry, so that for any $x_{1}, x_{2}$ and $k, u_{1}\left(x_{1}, x_{2}, k\right)=u_{2}\left(x_{2}, x_{1}, k\right)$. For $x \in X$ and $k$, we define $U(x, k)=u_{i}(x, x, k) .^{5}$

We make the following assumptions, which capture some features of Bertrand competition. The stage game may as well be called games with proportional temptations.

Assumption 1 The state of each period follows a common probability distribution, and it is independent over time. For any given period, the state of that period is $k$ with probability $p_{k} \in(0,1)$.

Assumption 2 For any $k, \Delta_{k} \equiv \max _{x \in X} U(x, k)$ exists, and $\Delta_{k}>0$ holds.

\footnotetext{
${ }^{2}$ For example, suppose that the per-market expected profit under full collusion is 1 . Then the limit result in [3] is consistent with a conclusion that the MCP is $M-\sqrt{M}$, when the number of markets is $M$. Our limit result in terms of the total profits reveals that this conclusion is impossible.

${ }^{3}$ In [2] and [4], the stage game is a prisoners' dilemma. Since our stage game is quite similar to their games, the main difference among those models is attributed to the players' monitoring ability.

${ }^{4} \mathrm{An}$ extension to the case of three or more players is straightforward.

${ }^{5}$ Due to symmetry, $U(x, k)$ does not depend on choice of $i$.
} 
Assumption 3 There exists $K>1$ such that for any $k$ and any $u \in\left[0, \Delta_{k}\right], x \in X$ exists such that

$$
U(x, k)=u, \quad \sup _{y \in X} u_{1}(y, x, k)=K u .
$$

Moreover, for any $k$, any $u \in\left(0, \Delta_{k}\right]$, and any $x^{\prime} \in X$ such that $U\left(x^{\prime}, k\right)=u$, we have $\sup _{y} u_{1}\left(y, x^{\prime}, k\right) \geq K u$.

Assumption 4 For each $k, \min _{x \in X} \sup _{y \in X} u_{1}(y, x, k)$ exists and equals zero. Moreover, the normal form game whose payoff function is given by $u_{i}\left(x_{1}, x_{2}, k\right)$ for each player $i$ has a unique Nash equilibrium payoff pair.

Assumption 5 We have $\Delta_{0} \leq \Delta_{1} \leq \cdots \leq \Delta_{M}$, and $\Delta_{0}<\Delta_{M}$.

Assumption 1 states that the states are i.i.d. over time. Assumption 2 guarantees existence of a maximum symmetric action pair payoff under any state, which is the value of full cooperation under that state. Together with Assumption 4, the maximum is greater than each player's minmax value given the state. ${ }^{6}$

Assumption 3 is the assumption of proportional temptations. It first states that given a state $k$, any payoff between the maximum symmetric action pair payoff and the minmax value is attained by some symmetric action pair $(x, x)$. Further, each player finds $x$ suboptimal against $x$ unless $U(x, k)=0$, and he can obtain either exactly or approximately $K$ times of $U(x, k) .{ }^{7}$ Note that the coefficient $K$ is independent of $k$. For $k$ and $u \in\left[0, \Delta_{k}\right]$, let $x(u ; k)$ be an element of $X$ satisfying (1). Assumption 3 also states that any other symmetric action pair $\left(x^{\prime}, x^{\prime}\right)$ with a payoff $u \in\left(0, \Delta_{k}\right]$ under state $k$ does not give each player a smaller temptation than $x(u ; k)$; we have $\sup _{y} u_{1}\left(y, x^{\prime}, k\right) \geq K u$.

Evaluating (1) at $u=0$, we see that $(x(0 ; k), x(0 ; k))$ is a Nash equilibrium of the game whose payoff function is $u_{i}\left(x_{1}, x_{2}, k\right)$ for each player $i$, and each player's equilibrium payoff is 0 . Assumption 4 states that it is the only Nash equilibrium payoff and the minmax value of that game. ${ }^{8}$ Since we will exclude randomized actions, the minmax value is defined by pure actions. Finally, Assumption 5 states that the states are ordered so that the values of full cooperation are nondecreasing, and that the values are not constant.

Let us denote this stage game by $G$. $G$ has a unique Nash equilibrium payoff pair $(0,0)$, and it is an equilibrium for each player to play $x(0 ; k)$ if the state is $k$.

We provide three examples of games satisfying Assumptions 2-5.

Example 1 (Bertrand oligopoly) Let $X=[0, \bar{p}]$ with $\bar{p}>0$. For each $k$, let

$$
u_{1}\left(x_{1}, x_{2}, k\right)= \begin{cases}x_{1} D\left(x_{1} ; k\right) & \text { if } x_{1}<x_{2}, \\ \frac{1}{2} x_{1} D\left(x_{1} ; k\right) & \text { if } x_{1}=x_{2}, \\ 0 & \text { if } x_{1}>x_{2},\end{cases}
$$

\footnotetext{
${ }^{6}$ Precisely speaking, the value is the minimum of suprema, but we abuse terminology and call it the minmax value.

${ }^{7}$ While (1) is stated in terms of player 1 , the counterpart for player 2 also holds by symmetry.

${ }^{8}$ This is consistent with multiple Nash equilibria, because more than one $x$ may satisfy (1) at $u=0$.
} 
where $D(\cdot ; k)$ is continuous and nonincreasing. We also assume $D(0 ; k)>D(\bar{p} ; k)=0 . u_{2}$ is derived from $u_{1}$ by symmetry. This is a standard model of Bertrand duopoly, where the costs are assumed to be zero for simplicity.

We have

$$
U(x, k)=\frac{1}{2} x D(x ; k) .
$$

By assumption, $\Delta_{k}=\max _{x \in X} U(x, k)$ exists, and $\Delta_{k}>0$. Assumption 2 thus holds.

For $u \in\left[0, \Delta_{k}\right]$, let $x(u ; k)$ be the smallest $x \in X$ such that $U(x, k)=u$. Since $U(x, k)$ is continuous in $x, x(u ; k)$ indeed exists. By the definition of $x(u ; k)$, we have $x D(x ; k)<2 u$ for any $x<x(u ; k)$. Hence by continuity,

$$
\sup _{y \in X} u_{1}(y, x(u ; k), k)=2 u \text {. }
$$

Furthermore, for any $x$ such that $U(x, k)=u$, we have $x \geq x(u ; k)$. Since $u_{1}\left(x_{1}, x_{2}, k\right)$ is nondecreasing in $x_{2}$, it holds that

$$
\sup _{y \in X} u_{1}(y, x, k) \geq \sup _{y \in X} u_{1}(y, x(u ; k), k)=2 u .
$$

Since $u \in\left[0, \Delta_{k}\right]$ is arbitrary, (2) and (3) imply that Assumption 3 holds for $K=2$.

In the normal-form game with player $i$ 's payoff function being $u_{i}\left(x_{1}, x_{2}, k\right)$, its Bertrand structure immediately means that each firm's equilibrium profit is zero, and this equals its minmax value. Thus Assumption 4 is satisfied.

Finally, Assumption 5 holds if we assume $D(p ; k)>D(p ; k-1)$ for any $k \geq 1$ and any $p<\bar{p}$. However, it holds under much weaker assumptions. While it does not hold if we just assume $D(p ; k) \geq D(p ; k-1)$ for any $k \geq 1$ and any $p$ (then it is possible that $\left.\Delta_{0}=\Delta_{1}=\cdots=\Delta_{M}\right)$, it will hold under suitable strengthening of it.

Example 2 (first-price auctions) Let $X=[0, \infty)$. For each $k$, let

$$
u_{1}\left(x_{1}, x_{2}, k\right)= \begin{cases}v_{k}-x_{1} & \text { if } x_{1}>x_{2} \text { and } x_{1} \geq r_{k} \\ \frac{1}{2}\left(v_{k}-x_{1}\right) & \text { if } x_{1}=x_{2} \geq r_{k} \\ 0 & \text { if } x_{1}<\min \left\{r_{k}, x_{2}\right\}\end{cases}
$$

where $v_{k}>r_{k} \geq 0 . u_{2}$ is derived from $u_{1}$ by symmetry. This is a first-price auction where two buyers' common valuation is $v_{k}$ and the reserve price is $r_{k}$ under state $k$.

We have

$$
U(x, k)= \begin{cases}\frac{1}{2}\left(v_{k}-x\right) & \text { if } x \geq r_{k} \\ 0 & \text { if } x<r_{k}\end{cases}
$$

It is easy to see that $\Delta_{k}=\left(v_{k}-r_{k}\right) / 2>0$. Assumption 2 is therefore satisfied.

For any $k$ and any $u \in\left[0, \Delta_{k}\right]$, a solution of $U(x, k)=u$ is $x=v_{k}-2 u$, and it is a unique solution if $u>0$. Since we also have

$$
\sup _{y \in X} u_{1}\left(y, v_{k}-2 u, k\right)=2 u
$$


Assumption 3 holds for $K=2$.

For each $k$, the normal form game with player $i$ 's payoff function being $u_{i}\left(x_{1}, x_{2}, k\right)$ has Bertrand structure. Hence, any equilibrium payoff is zero, which equals each buyer's minmax value. Assumption 4 is therefore satisfied. Finally, Assumption 5 is satisfied if we assume $v_{k}-r_{k}$ is nondecreasing and $v_{0}-r_{0}<v_{M}-r_{M}$.

Example 3 (linear payoffs and multiplicative shocks) Let $X=[0,1]$, and let

$$
u_{1}\left(x_{1}, x_{2}, k\right)=\theta_{k}\left(\alpha x_{2}-x_{1}\right), \quad u_{2}\left(x_{1}, x_{2}, k\right)=\theta_{k}\left(\alpha x_{1}-x_{2}\right)
$$

for each $k$, where $\theta_{k}>0$ and $\alpha>1$. Therefore we have $U(x, k)=\theta_{k}(\alpha-1) x$, and $\Delta_{k}=\theta_{k}(\alpha-1)>0$. Hence Assumption 2 is satisfied.

For any $u \in\left[0, \Delta_{k}\right]$, the unique solution of $U(x, k)=u$ is $x=u / \Delta_{k}$. Since

$$
\sup _{y} u_{1}\left(y, \frac{u}{\Delta_{k}}, k\right)=u_{1}\left(0, \frac{u}{\Delta_{k}}, k\right)=\frac{\alpha u}{\alpha-1},
$$

(1) holds for $K=\alpha /(\alpha-1)>1$. Assumption 3 is therefore satisfied.

In the normal-form game with player $i$ 's payoff function being $u_{i}\left(x_{1}, x_{2}, k\right), 0$ is a dominant action and minmaxes the other player. Hence 0 is indeed the Nash equilibrium payoff and the minmax value, which guarantees Assumption 4. Finally, Assumption 5 holds if we assume $\theta_{0} \leq \theta_{1} \leq \cdots \leq \theta_{M}$ with $\theta_{0}<\theta_{M}$.

This is a game with very simple structure, but it includes moral hazard in teams and public goods provision with linear technology as examples.

Note that in all these examples, $\Delta_{k}$ is the maximum of the stage payoff sum, even if we take asymmetric action pairs into account under state $k$.

The players play $G$ in periods $0,1,2, \ldots$ Each player knows the state of each period at the beginning of that period, but does not know the state of any future period until that period arrives. We also assume perfect monitoring. Namely, the players can observe the other players' past actions, together with all past states. In the present paper, we limit attention to pure strategies. Thus each player $i$ 's strategy of this repeated game is a function which maps a history at each period $t$, consisting of $\left(x_{1}(\tau), x_{2}(\tau)\right)_{\tau=0}^{t-1}$ and $(k(\tau))_{\tau=0}^{t}$, where $x_{i}(\tau)$ is player $i$ 's action in period $\tau$ and $k(\tau)$ is the realized state of period $\tau$, to an element of $X$. Note that a history at period $t$ includes the state of period $t$. Given a strategy pair, player $i$ 's expected payoff of the repeated game is:

$$
(1-\delta) E\left[\sum_{t=0}^{\infty} \delta^{t} u_{i}\left(x_{1}(t), x_{2}(t), k(t)\right)\right]
$$

where $\delta \in(0,1)$ is a common discount factor, and the expectation is taken with respect to the states of the entire periods.

Let us denote this infinitely repeated game by $G(\delta)$. A strategy pair is symmetric if at no history the players' actions are different. Our solution concept for $G(\delta)$ is the most cooperative equilibrium (MCE), which we define as the equilibria attaining the largest expected payoff among all symmetric subgame perfect equilibria of $G(\delta)$. The MCE expected payoff is called the most cooperative payoff (MCP). 


\section{Characterization of the MCP}

This section fully characterizes the MCP of $G(\delta)$ for any $\delta$. The following function defined on $[0, \infty)$ is useful.

$$
g(b)=b-\sum_{k=0}^{M} p_{k} \min \left\{\Delta_{k}, \frac{\delta b}{(1-\delta)(K-1)}\right\} .
$$

The idea of this function is closely related to the following strategy pair with the payoff target $B$.

(i) In period 0 with state $k(0)$, each player plays $x\left(\min \left\{\Delta_{k(0)}, B\right\} ; k(0)\right)$.

(ii) In period $t(t \geq 1)$, given the corresponding history $(a(\tau))_{\tau=0}^{t-1}$ and $(k(\tau))_{\tau=0}^{t}$,

(a) if $x_{1}(\tau)=x_{2}(\tau)=x\left(\min \left\{\Delta_{k(\tau)}, B\right\} ; k(\tau)\right)$ for any $\tau \leq t-1$, then each player plays $x\left(\min \left\{\Delta_{k(t)}, B\right\} ; k(t)\right)$, and

(b) otherwise, each player plays $x(0 ; k(t))$.

On the path of this strategy pair, the players fully cooperate under any state whose payoff from full cooperation is below the target $B$, and they play actions achieving the target under any state whose payoff from full cooperation exceeds it. At any history on the path whose current state is $k$, the stage payoff under this strategy pair is $\min \left\{\Delta_{k}, B\right\}$. From Assumption 3, the incremental stage payoff from a deviation at such a history is at most $(K-1) B$. Since the continuation payoff given a deviation is zero, this strategy pair is an equilibrium if

$$
(1-\delta)(K-1) B \leq \delta \sum_{k=0}^{M} p_{k} \min \left\{\Delta_{k}, B\right\} .
$$

Hence, for any $b$ such that $g(b) \leq 0$, the corresponding strategy pair with the payoff target $B=\delta b /\{(1-\delta)(K-1)\}$ is an equilibrium. Note that $g(b)$ measures the gain from the most profitable deviation.

Note that $g$ is continuous and convex. ${ }^{9}$ Since $g(0)=0$ and $g(b)>0$ for any large $b$, the set of all $b$ 's with $g(b) \leq 0$ has the form $\left[0, b^{*}\right]$. Note that $b^{*}$ depends on $\delta$, and we have $g\left(b^{*}\right)=0$. Hence, the corresponding strategy pair with the payoff target $B^{*}=\delta b^{*} /\{(1-\delta)(K-1)\}$ is an equilibrium, and its payoff is $b^{*}$.

Proposition 1 For any $\delta, b^{*}$ is the $M C P$ of $G(\delta)$.

Proof. Fix an MCE of $G(\delta)$, and let $b$ be its payoff. For each $k$, let $B_{k}$ be the stage-game payoff of the initial period when its state is $k$. Define $\hat{b}=\sum_{k=0}^{M} p_{k} B_{k}$. Since $b$ is the MCP, we have $b \leq \hat{b}$ (otherwise, the expected continuation equilibrium payoff is greater than $b$, a contradiction).

From Assumption 3 and the definition of $b$, the equilibrium condition implies

$$
(1-\delta)(K-1) B_{k} \leq \delta b \leq \delta \hat{b}
$$

\footnotetext{
${ }^{9}$ It is piecewise linear, and its slope is nondecreasing.
} 
for any $k$. By the definition of $B_{k}$ 's, we have $B_{k} \leq \Delta_{k}$ for any $k$. Combining this with (4) yields

$$
B_{k} \leq \min \left\{\Delta_{k}, \frac{\delta \hat{b}}{(1-\delta)(K-1)}\right\}
$$

for any $k$. Taking expectations of $(5)$, we obtain $g(\hat{b}) \leq 0$. By the definition of $\hat{b}$ and $b^{*}$, we have $b \leq \hat{b} \leq b^{*}$. Since we have seen that $b^{*}$ is an equilibrium payoff, we have $b=b^{*}$ as desired.

Q.E.D.

In order to examine the MCP more thoroughly, define $\underline{\delta}$ and $\bar{\delta}$ as follows.

$$
\underline{\delta}=\frac{K-1}{K}, \quad \bar{\delta} \equiv \frac{(K-1) \Delta_{M}}{(K-1) \Delta_{M}+\sum_{k=0}^{M} p_{k} \Delta_{k}} .
$$

It is easy to see that $\underline{\delta}<\bar{\delta}$.

We have four cases to consider. First, if $\delta<\underline{\delta}, g(b)>0$ for any $b>0$. Hence, the $\mathrm{MCP}$ is zero. The discount factor is so small that any positive payoff cannot be sustained as an equilibrium, and the repeated play of a symmetric stage equilibrium is an MCE.

Second, if $\delta=\underline{\delta}, g\left(\Delta_{0}\right)=0$ and $g(b)>0$ for any $b>\Delta_{0}$. Hence, the MCP is $\Delta_{0}$. The MCE attains a positive payoff, but it plays as if the current state was zero even under other states. The players thus suffer from burden of payoff fluctuations.

Third, if $\delta \geq \bar{\delta}, g(b) \leq 0$ if and only if $b \leq \sum_{k=0}^{M} p_{k} \Delta_{k}$. Hence, the MCP is the expected value of the payoffs from full cooperation, the best possible payoff of this game. The discount factor is so large that the players can fully cooperate.

Finally, if $\delta \in(\underline{\delta}, \bar{\delta})$, the MCP belongs to the interval $\left(\Delta_{0}, \sum_{k=0}^{M} p_{k} \Delta_{k}\right)$. The players partially cooperate, and due to the above observations, the MCP is continuous and strictly increasing on the range $[\underline{\delta}, \bar{\delta}]$. Thus, more patience reduces the loss from fluctuated payoffs.

In all cases, the MCE has a payoff target $B$. Namely, in its cooperative phase, the players play a symmetric action pair whose payoff is $\min \left\{\Delta_{k}, B\right\}$ under state $k$. When the MCE only attains partial cooperation, they must give up full cooperation under higher states. ${ }^{10}$ This is an efficient way to provide incentives, because the incentive conditions under higher states are duplicated.

If $\delta>\underline{\delta}$, we have $g\left(\Delta_{0}\right)<0$. Since $g$ is convex, this implies that $b>\Delta_{0}$ such that $g(b)=0$ is unique. Hence, it suffices to solve $g(b)=0$, as the following corollary shows.

Corollary 1 If $\delta>\underline{\delta}$ and $g(b)=0$ for $b>\Delta_{0}$, then $b$ is the $M C P$ of $G(\delta)$.

\section{Multimarket Contact}

This section applies the results in the previous section to Bertrand price competition with demand fluctuations, in order to examine effects of multimarket contact. We first describe the environment and then set it up as a game satisfying all assumptions.

There are $M$ ex ante identical markets, and in each market two identical firms compete in price. Each market is subject to demand fluctuations, depending on which it is either

\footnotetext{
${ }^{10}$ If the discount factor is so small that no cooperation is possible, the target is zero. If it is so large that full cooperation is possible, the target can be set so high that it never binds.
} 
in high demand or low demand. The demands are independent across the markets and over time. The probability that a given market is in high demand is $\mu \in(0,1)$. We assume that each market is associated with the Bertrand price competition game we described in Example 1, except that we now have only two states; ones corresponding to high and low demands, respectively. Let $\pi_{H}\left(\pi_{L}\right.$, respectively) be each firm's profit under full collusion (the value corresponding to $\Delta_{k}$ ), when the demand is high (low). We assume $\pi_{H}>\pi_{L}>0$. Define $\bar{\pi}=\mu \pi_{H}+(1-\mu) \pi_{L}$, which is the expected value of full collusion per market.

At the beginning of each period, the firms learn which of the $M$ markets are in high demand in that period. Given that, they decide prices in all markets. Formally, the number of states is $2^{M}$, and the set of actions is $X=[0, \bar{p}]^{M}$. Since each market satisfies all assumptions with $K=2$, it is easy to see that this environment also satisfies all assumptions with $K=2$. Note that the maximum symmetric action pair profit for a given state depends only on the number of high-demand markets. Thus, for $l \in\{0,1, \ldots, M\}$, let $\Delta_{l}$ be the maximum symmetric action pair profit when $l$ markets are in high demand:

$$
\Delta_{l}=l \pi_{H}+(M-l) \pi_{L}>0 .
$$

Let us denote the repeated game with this stage game by $G(\delta, M)$.

The results in the previous section directly apply to this model. Note that

$$
\underline{\delta}=\frac{1}{2}, \quad \bar{\delta}=\frac{\pi_{H}}{\pi_{H}+\bar{\pi}} .
$$

Let $b_{M}^{*}$ be the MCP of $G(\delta, M)$. Define $\beta_{M}^{*}=b_{M}^{*} / M$ as the per-market MCP of $G(\delta, M)$. The following observations immediately follow from the analysis in the previous section.

(I) If $\delta<\underline{\delta}, b_{M}^{*}=\beta_{M}^{*}=0$ for any $M$. Therefore any collusion is impossible, regardless of the number of markets. In this case, multimarket contact does not help at all.

(II) If $\delta=\underline{\delta}, b_{M}^{*}=M \pi_{L}$ and $\beta_{M}^{*}=\pi_{L}$ for any $M$. That is, independent of the number of markets, the MCP equals the value of full collusion when all markets are in low demand. This is another case where multimarket contact does not help.

(III) If $\delta \geq \bar{\delta}, b_{M}^{*}=M \bar{\pi}$ and $\beta_{M}^{*}=\bar{\pi}$ for any $M$. Namely, the firms can fully collude regardless of the number of markets. In this case, multimarket contact does not matter.

The remaining case is $\underline{\delta}<\delta<\bar{\delta}$. Since the profit from full collusion given a state depends only on the number of high-demand markets, the condition for the MCE $\left(g\left(b_{M}^{*}\right)=\right.$ 0) can be rewritten as follows.

$$
b_{M}^{*}=\sum_{k=0}^{M} p_{k} \min \left\{\Delta_{k}, \frac{\delta b_{M}^{*}}{1-\delta}\right\}, \quad \therefore \beta_{M}^{*}=\sum_{k=0}^{M} p_{k} \min \left\{\frac{\Delta_{k}}{M}, \frac{\delta \beta_{M}^{*}}{1-\delta}\right\},
$$

where $p_{k}$ is the probability that $k$ markets are in high demand. In what follows, we examine how the MCP and the per-market MCP vary with $M$.

Proposition 2 Let $\delta \in(\underline{\delta}, \bar{\delta})$. For any $M \geq 1$, we have $\beta_{M}^{*} \leq \beta_{M+1}^{*}$, and the equality 
holds if and only if there exists $\hat{k} \in\{1,2, \ldots, M-1\}$ such that

$$
\frac{\delta b_{M}^{*}}{1-\delta}=\Delta_{\hat{k}}
$$

Proof. See Appendix A.

Proposition 2 reveals that for relatively patient firms which can only partially collude, adding one more market never reduces the per-market MCP and increases it except when the condition (7) holds. For a fixed $M$, we have seen that the MCP is increasing on the range $(\underline{\delta}, \bar{\delta})$. Therefore, $(7)$ holds only at $M-1$ discount factors. In other words, adding one more market facilitates collusion for almost all discount factors on the range.

If $M=1$, the condition (7) is vacuous. Further, suppose (7) holds under some $M$ and $\hat{k} \in\{1,2, \ldots, M-1\}$, and therefore we have $\beta_{M}^{*}=\beta_{M+1}^{*}$. Then it follows that

$$
\hat{k} \pi_{H}+(M+1-\hat{k}) \pi_{L}<\frac{\delta b_{M+1}^{*}}{1-\delta}<(\hat{k}+1) \pi_{H}+(M-\hat{k}) \pi_{L}
$$

From these observations we have the following corollary.

Corollary 2 Let $\delta \in(\underline{\delta}, \bar{\delta})$. Then we have (i) $\beta_{1}^{*}<\beta_{2}^{*}$, and (ii) for any $M \geq 1$ and any $M^{\prime} \geq M+2, \beta_{M}^{*}<\beta_{M^{\prime}}^{*}$

Why do these results hold? Recall that the MCE has a profit target, and let $B$ be that of the MCE under $M$ markets. In the proof, we show that under $M+1$ markets, a strategy pair whose profit target is $(M+1) B / M$ has a per-market profit not smaller than the per-market MCP under $M$ markets. Since the per-market profit target remains the same (and equals $B / M$ ), this strategy pair gives the firms the same per-market temptation to deviate. Hence, this strategy pair is an equilibrium, and adding one more market is shown to (weakly) improve the per-market MCP.

Suppose $k$ out of the $M+1$ markets are in high demand. If we ignore the $(M+1)$ th market, we can compare with the case of $M$ markets. Conditional on this event, the incremental per-market profit of having one more market is

$$
\min \left\{\frac{k \pi_{H}+(M+1-k) \pi_{L}}{M+1}, \frac{B}{M}\right\}-\frac{k \min \left\{\frac{\Delta_{k-1}}{M}, \frac{B}{M}\right\}+(M+1-k) \min \left\{\frac{\Delta_{k}}{M}, \frac{B}{M}\right\}}{M+1} .
$$

This is because the $(M+1)$-th market is in high demand with probability $k /(M+1)$, conditional on that there are $k$ high-demand markets.

If the new strategy pair prescribes full collusion given $k$ high-demand markets, the MCE under $M$ markets also prescribes full collusion given $k-1$ high-demand markets. Hence, the incremental profit is zero if it also prescribes full collusion given $k$ high-demand markets. However, this is not always the case. The MCE under $M$ markets may prescribe partial collusion (to the profit target level) given $k$ high-demand markets. The incremental profit is positive in this case. Note that this happens at most one $k$; the smallest $k$ at which the MCE prescribes partial collusion.

Instead, if $k$ prescribes partial collusion given $k$ high-demand markets, the MCE under $M$ markets also prescribes partial collusion given $k$ high-demand markets. Hence, the incremental profit is zero if it also prescribes partial collusion given $k-1$ high-demand markets. However, this is not always the case. The MCE under $M$ markets may prescribe 
full collusion given $k-1$ high-demand markets. The incremental profit is positive in this case. Note that this happens at most one $k$; again, the smallest $k$ at which the MCE prescribes partial collusion.

To sum up, the incremental profit can be positive only at one $k$, and is zero at all other $k$ 's. A special case is where the condition (7) holds. In this special case, the incremental profit is zero even at that $k$, so that adding one market does not improve the per-market MCP. In all other cases, the per-market MCP increases.

The next result is about the MCP in the limit, showing that for relatively patient firms, the difference between the expected profit under full collusion and the MCP converges to zero if the number of markets goes to infinity. Namely, the collusion-deterrence effects of demand fluctuations completely vanish in the limit.

Proposition 3 Let $\delta \in(\underline{\delta}, \bar{\delta})$, and $f i x \varepsilon>0$ arbitrarily. Then there exists $\underline{M}$ such that for any $M \geq \underline{M}, b_{M}^{*}>\bar{\pi} M-\varepsilon$. In other words, $\lim _{M \rightarrow \infty}\left(\bar{\pi} M-b_{M}^{*}\right)=0$.

Proof. See Appendix B.

From the proof of Proposition 3, we see that the MCE when $M$ is large enough sets a profit target greater than $M\left\{\hat{\mu} \pi_{H}+(1-\hat{\mu}) \pi_{L}\right\}$, where $\hat{\mu}>\mu$. That is, the per-market target exceeds the average profit from full collusion. By the law of large numbers, the probability that the fraction of high-demand markets is greater than $\hat{\mu}$ converges to zero, if the number of markets goes to infinity. In fact, the convergence is so fast that it converges to zero even if it is multiplied by $M$. This implies that the firms can fully collude except upon an event with a negligible probability, and that the expected efficiency loss due to demand fluctuations is also negligible. Hence, full collusion is approximately attained.

While Proposition 3 shows that $\bar{\pi} M-b_{M}^{*}$ converges to zero as $M \rightarrow \infty$, it is not monotone. A good example is when (7) holds. Then we have $\beta_{M}^{*}=\beta_{M+1}^{*}$, and therefore

$$
\bar{\pi}(M+1)-b_{M+1}^{*}-\left(\bar{\pi} M-b_{M}^{*}\right)=\bar{\pi}-\frac{b_{M+1}^{*}}{M+1}>0
$$

where the last inequality follows because full collusion is not sustainable for any $\delta \in(\underline{\delta}, \bar{\delta})$.

We present some numerical analysis. Let us suppose $\pi_{H}=15, \pi_{L}=10$, and $\mu=0.6$, which imply that $\bar{\pi}=13$ and $\bar{\delta}=15 / 28 \simeq 0.5357$. Table 1 exhibits the per-market MCP under various numbers of markets and the minimum number of markets which makes $b_{M}^{*}$ differ from the expected profit under full collusion $(M \bar{\pi})$ by less than one, for various discount factors. For instance, if $\delta=0.52$, the case where the firms are relatively patient, having three markets allows them to obtain more than 38 , while the expected profit under full collusion is $13 \times 3=39$. If they become less patient, the MCP decreases given the number of markets, and they need a much greater number of markets in order to sustain outcomes very close to full collusion. The case of $\delta=125 / 246$ is interesting in the sense that the condition ( 7 ) holds when $M=2$. Note that $\beta_{2}^{*}=\beta_{3}^{*}$ in this case.

We conclude by emphasizing importance of the assumption that the demands are independent across all markets. ${ }^{11}$ Namely, all demand shocks are idiosyncratic to each market. To the extent that common macro shocks are present, the Rotemberg-Saloner effect (or collusion-deterrence due to fluctuated demands) would not be "averaged out."

\footnotetext{
${ }^{11}$ We are thankful to an anonymous referee for suggesting the arguments in this paragraph.
} 
Table 1: Numerical Analysis for $\pi_{H}=15, \pi_{L}=10$, and $\mu=0.6$

\begin{tabular}{|c|c|c|c|c|c|}
\hline & $\delta=0.52$ & $\delta=0.51$ & $\delta=\frac{125}{246}$ & $\delta=0.505$ & $\delta=0.501$ \\
\hline$\beta_{2}^{*}$ & 12.459 & 12.154 & 12.1 & 11.188 & 10.212 \\
$\beta_{3}^{*}$ & 12.742 & 12.287 & 12.1 & 11.802 & 10.615 \\
$\beta_{5}^{*}$ & 12.922 & 12.636 & 12.586 & 12.223 & 11.360 \\
$\min \left\{M: M \bar{\pi}-b_{M}^{*}<1\right\}$ & 3 & 26 & 49 & 201 & 11566 \\
\hline
\end{tabular}

This observation casts some doubts about validity of our limit result when the number of markets goes to infinity (Proposition 3). Imagine a process of adding new markets from a pool of potential markets. As long as the number of markets is small, it may not be difficult to choose them so that all demand shocks are independent. As more and more markets are added, however. such independence may be hard to maintain. Consequently, our model might be most relevant when there are not so many markets. In this case, our result on the effect of adding one more market (Proposition 2) is most important. ${ }^{12}$

\section{A Appendix: Proof of Proposition 2}

Fix $\delta \in(\underline{\delta}, \bar{\delta})$ and $M \geq 1$. Let $p_{l}(l \in\{0,1, \ldots, M\})$ be the probability that $l$ out of $M$ markets are in high demand, and let $q_{l}(l \in\{0,1, \ldots, M+1\})$ be the probability that $l$ out of $M+1$ markets are in high demand. Formally,

$$
p_{l}=\frac{M !}{l !(M-l) !} \mu^{l}(1-\mu)^{M-l}, \quad q_{l}=\frac{(M+1) !}{l !(M+1-l) !} \mu^{l}(1-\mu)^{M+1-l} .
$$

Note that from these, the following equations hold for any $l \in\{0, \ldots, M\}$;

$$
\frac{p_{l-1} \mu \Delta_{l-1}+p_{l}(1-\mu) \Delta_{l}}{M}=q_{l} \frac{l \pi_{H}+(M+1-l) \pi_{L}}{M+1}, \quad p_{l} \mu+p_{l+1}(1-\mu)=q_{l+1} .
$$

Here, the convention is that $p_{-1}=p_{M+1}=0$.

Since $\underline{\delta}<\delta<\bar{\delta}$, we have $\pi_{L}<\delta \beta_{M}^{*} /(1-\delta)<\pi_{H}$. Thus there exists $\hat{k} \in\{0,1, \ldots, M\}$ such that

$$
\frac{\hat{k} \pi_{H}+(M+1-\hat{k}) \pi_{L}}{M+1}<\frac{\delta \beta_{M}^{*}}{1-\delta} \leq \frac{(\hat{k}+1) \pi_{H}+(M-\hat{k}) \pi_{L}}{M+1} .
$$

Recall that $\Delta_{l}=l \pi_{H}+(M-l) \pi_{L}$. Thus, (9) implies

$$
\frac{\Delta_{\hat{k}-1}}{M}<\frac{\delta \beta_{M}^{*}}{1-\delta}<\frac{\Delta_{\hat{k}+1}}{M} .
$$

\footnotetext{
${ }^{12}$ Further, the firms may want to divide existing markets into "submarkets," in order to exploit the effects of multimarket contact. Of course, the performance of this strategy crucially depends on the nature of demand shocks across the submarkets. They may even want to design the length of a period, in an effort to control the flow of demand shocks.
} 
Let us rearrange (6) by using (10):

$$
\begin{aligned}
\beta_{M}^{*} & =\left(\sum_{l=0}^{\hat{k}-1} \frac{p_{l} \Delta_{l}}{M}\right)+p_{\hat{k}} \min \left\{\frac{\Delta_{\hat{k}}}{M}, \frac{\delta \beta_{M}^{*}}{1-\delta}\right\}+\left(\sum_{l=\hat{k}+1}^{M} p_{l}\right) \frac{\delta \beta_{M}^{*}}{1-\delta} \\
& \leq\left(\sum_{l=0}^{\hat{k}-1} \frac{p_{l} \Delta_{l}}{M}\right)+\frac{p_{\hat{k}}(1-\mu) \Delta_{\hat{k}}}{M}+\left(p_{\hat{k}} \mu+\sum_{l=\hat{k}+1}^{M} p_{l}\right) \frac{\delta \beta_{M}^{*}}{1-\delta} .
\end{aligned}
$$

Substituting (8) into (11) and using (9), we obtain

$$
\beta_{M}^{*} \leq \sum_{l=0}^{M+1} q_{l} \min \left\{\frac{l \pi_{H}+(M+1-l) \pi_{L}}{M+1}, \frac{\delta \beta_{M}^{*}}{1-\delta}\right\} .
$$

Let us consider the counterpart of (6) for the case of $M+1$ markets:

$$
\beta_{M+1}^{*}=\sum_{l=0}^{M+1} q_{l} \min \left\{\frac{l \pi_{H}+(M+1-l) \pi_{L}}{M+1}, \frac{\delta \beta_{M+1}^{*}}{1-\delta}\right\} .
$$

If (7) does not hold, (11) holds with strict inequality and so does (12). Comparing this with (13), we obtain $\beta_{M}^{*}<\beta_{M+1}^{*}$ as desired. If (7) holds, note that this occurs only if $1 \leq \hat{k} \leq M-1$ because of $M \pi_{L}<\delta b_{M}^{*} /(1-\delta)<M \pi_{H}$. Now (12) holds with equality. Comparing this with (13) and applying Corollary 1 , we obtain $\beta_{M+1}^{*}=\beta_{M}^{*}$ as desired.

\section{B Appendix: Proof of Proposition 3}

In what follows, let $p_{k}^{M}$ be the probability that $k$ out of $M$ markets are in high demand. Fix $\delta \in(\underline{\delta}, \bar{\delta})$ and $\varepsilon>0$. Since $\delta>\underline{\delta}$, a rational number $\hat{\mu} \in(\mu, 1)$ exists such that

$$
\frac{\delta}{1-\delta} \bar{\pi}=\frac{\delta}{1-\delta}\left\{\mu \pi_{H}+(1-\mu) \pi_{L}\right\}>\hat{\mu} \pi_{H}+(1-\hat{\mu}) \pi_{L}
$$

It then follows that

$$
\frac{\delta}{1-\delta}(\bar{\pi} M-\varepsilon)>M\left\{\hat{\mu} \pi_{H}+(1-\hat{\mu}) \pi_{L}\right\}
$$

for all large $M$. Therefore, for all large $M$ we have

$$
\begin{aligned}
\sum_{l=0}^{M} p_{l}^{M} \min \left\{\Delta_{l}, \frac{\delta(\bar{\pi} M-\varepsilon)}{1-\delta}\right\} & \geq \sum_{l=0}^{M} p_{l}^{M} \min \left[\Delta_{l}, M\left\{\hat{\mu} \pi_{H}+(1-\hat{\mu}) \pi_{L}\right\}\right] \\
& =\sum_{l=0}^{M} p_{l}^{M}\left[\Delta_{l}+\min \left\{0,(M \hat{\mu}-l)\left(\pi_{H}-\pi_{L}\right)\right\}\right] \\
& =\bar{\pi} M-\left(\pi_{H}-\pi_{L}\right) \sum_{l=0}^{M} p_{l}^{M} \max \{0, l-M \hat{\mu}\}
\end{aligned}
$$


The proof is complete if we show that

$$
\lim _{M \rightarrow \infty} \sum_{l=0}^{M} p_{l}^{M} \max \{0, l-M \hat{\mu}\}=0 .
$$

This is because we then obtain

$$
\bar{\pi} M-\varepsilon<\sum_{l=0}^{M} p_{l}^{M} \min \left\{\Delta_{l}, \frac{\delta(\bar{\pi} M-\varepsilon)}{1-\delta}\right\}
$$

for all large $M$. Hence, the equilibrium condition at $\bar{\pi} M-\varepsilon$ (namely, $b(\bar{\pi} M-\varepsilon) \leq 0$ ) holds with strict inequality, so that Proposition 1 implies that $b_{M}^{*}>\bar{\pi} M-\varepsilon$ for all large $M$.

To prove (14), note that

$$
\sum_{l=0}^{M} p_{l}^{M} \max \{0, l-M \hat{\mu}\}=\sum_{l=\lceil M \hat{\mu}\rceil}^{M} p_{l}^{M}(l-M \hat{\mu})<(1-\hat{\mu}) M \sum_{l=\lceil M \hat{\mu}\rceil}^{M} p_{l}^{M},
$$

where $\lceil k\rceil$ is the smallest integer not less than $k$. Note also that

$$
\frac{p_{l+1}^{M}}{p_{l}^{M}}=\frac{(M-l) \mu}{(l+1)(1-\mu)}<\frac{(1-\hat{\mu}) \mu}{\hat{\mu}(1-\mu)} \equiv \kappa
$$

for any $l \geq\lceil M \hat{\mu}\rceil$. Since $\kappa<1$ from $\hat{\mu}>\mu$, we have

$$
(1-\hat{\mu}) M \sum_{l=\lceil M \hat{\mu}\rceil}^{M} p_{l}^{M}<\frac{1-\hat{\mu}}{1-\kappa} M p_{\lceil M \hat{\mu}\rceil}^{M} .
$$

Therefore, it suffices to prove that $M p_{\lceil M \hat{\mu}\rceil}^{M}$ goes to zero as $M \rightarrow \infty$.

Since $\hat{\mu}$ is rational, two natural numbers $y$ and $Y$ exist such that $\hat{\mu}=y / Y$. Let us consider the function $f(z)=z^{y}(1-z)^{Y-y}$. Note that $f$ is positive only when $z \in(0,1)$, and that the first-order condition on the range $(0,1)$ reduces to

$$
y(1-z)-(Y-y) z=0 .
$$

Therefore, $f$ is uniquely maximized at $z=y / Y=\hat{\mu}$. Hence there exists $\eta>0$ such that

$$
\left(\frac{\mu}{\hat{\mu}}\right)^{y}\left(\frac{1-\mu}{1-\hat{\mu}}\right)^{Y-y}<1-\eta \text {. }
$$

We also have

$$
\frac{(M+Y) p_{\lceil(M+Y) \hat{\mu}\rceil}^{M+Y}}{M p_{\lceil M \hat{\mu}\rceil}^{M}}=\frac{M+Y}{M} \cdot \frac{\left[\prod_{k=1}^{Y}(M+k)\right] \mu^{y}(1-\mu)^{Y-y}}{\left[\prod_{k=1}^{y}(\lceil M \hat{\mu}\rceil+k)\right]\left[\prod_{k=1}^{Y-y}(M-\lceil M \hat{\mu}\rceil+k)\right]}
$$

for each $M$, because $\lceil(M+Y) \hat{\mu}\rceil=\lceil M \hat{\mu}\rceil+y$.

Since $M \hat{\mu} \leq\lceil M \hat{\mu}\rceil<M \hat{\mu}+1$ for any $M,\lceil M \hat{\mu}\rceil / M$ goes to $\hat{\mu}$ as $M \rightarrow \infty$. Applying 
this to (16) and using (15), we have

$$
\lim _{M \rightarrow \infty} \frac{(M+Y) p_{\lceil(M+Y) \hat{\mu}\rceil}^{M+Y}}{M p_{\lceil M \hat{\mu}\rceil}^{M}}=\left(\frac{\mu}{\hat{\mu}}\right)^{y}\left(\frac{1-\mu}{1-\hat{\mu}}\right)^{Y-y}<1-\eta .
$$

Therefore, for any $z=1,2, \ldots, Y,(K Y+z) p_{\lceil(K Y+z) \hat{\mu}\rceil}^{K Y+z}$ goes to zero as $K \rightarrow \infty$. This proves that $M p_{\lceil M \hat{\mu}\rceil}^{M}$ goes to zero as $M \rightarrow \infty$.

\section{References}

[1] D. Bernheim, M. Whinston, Multimarket contact and collusive behavior, RAND J. Econ. 21 (1990) 1-26.

[2] H. Kobayashi, K. Ohta, Optimal collusion under imperfect monitoring in multimarket contact, Games Econ. Behav. 76 (2012) 636-647.

[3] J. Li, M. Powell, A note on the limits of multilateral cooperation, 2013, mimeo.

[4] H. Matsushima, Multimarket contact, imperfect monitoring and implicit collusion, J. Econ. Theory 98 (2001) 158-178.

[5] J. Rotemberg, G. Saloner, A supergame-theoretic model of price wars during booms, Amer. Econ. Rev. 76 (1986) 390-407.

[6] T. Sekiguchi, Multimarket contact under demand fluctuations: A limit result, 2013, Working Paper, No. E-52, Tokyo Center for Economic Research. 